\title{
Long non-coding RNA CASC2 inhibits breast cancer cell growth and metastasis through the regulation of the miR-96-5p/SYVN1 pathway
}

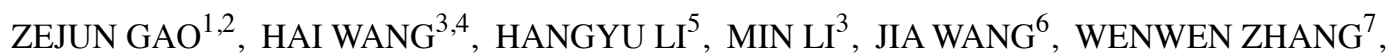 \\ XIUBIN LIANG ${ }^{3}$, DONGMING SU ${ }^{3,4}$ and JINHAI TANG ${ }^{1}$
}

\begin{abstract}
${ }^{1}$ Department of General Surgery, The First Affiliated Hospital with Nanjing Medical University, Nanjing, Jiangsu 210029;
${ }^{2}$ Department of General Surgery, Affiliated Hospital of Integrated Traditional Chinese and Western Medicine, Nanjing University of Chinese Medicine, Nanjing, Jiangsu 210028; ${ }^{3}$ Department of Pathology, Nanjing Medical University, Nanjing, Jiangsu 211166; ${ }^{4}$ Department of Pathology and Clinical Laboratory, Sir Run Run Hospital Affiliated with Nanjing Medical University, Nanjing, Jiangsu 211166; ${ }^{5}$ Department of General Surgery,

The Fourth Affiliated Hospital of China Medical University, Shenyang, Liaoning 110032; ${ }^{6}$ Department of General Surgery, The Second Affiliated Hospital with Dalian Medical University, Dalian, Liaoning 116027; ${ }^{7}$ Department of Clinical Laboratory, Children's Hospital of Nanjing Medical University, Nanjing, Jiangsu 210008, P.R. China
\end{abstract}

Received April 19, 2018; Accepted August 6, 2018

DOI: $10.3892 /$ ijo.2018.4522

\begin{abstract}
Cancer susceptibility candidate 2 (CASC2), a long non-coding RNA (lncRNA), has been demonstrated to be a tumor suppressor in several types of cancer. However, the role and mechanism of CASC 2 in breast cancer (BC) have not been investigated. In the present study, the expression and functions of CASC2 in BC were investigated. The expression of CASC2 was significantly decreased in $\mathrm{BC}$ tissues and cells compared with adjacent normal tissues and mammary epithelial cells, respectively. CASC2 overexpression inhibited the viability, migration and invasion, and elevated apoptosis of BC cells. In addition, CASC2 acted as a competing endogenous RNA for hsa-microRNA (miR)-96-5p and regulated the expression of its target gene, synoviolin (SYVN1). In miR-96-5p-overexpressed MDA-MB-231 cells, cell viability, migration and invasion was increased, and cell apoptosis was decreased, which was reversed by the upregulation of SYVN1. Taken together, the present study data indicated that decreased SYVN1 expression
\end{abstract}

Correspondence to: Professor Dongming $\mathrm{Su}$, Department of Pathology, Nanjing Medical University, 289 Longmian Avenue, Nanjing, Jiangsu 211166, P.R. China

E-mail: sudongming@njmu.edu.cn

Dr Jinhai Tang, Department of General Surgery, The First Affiliated Hospital with Nanjing Medical University, 300 Guangzhou Road, Nanjing, Jiangsu 210029, P.R. China

E-mail: jhtang@njmu.edu.cn

Key words: breast cancer, cancer susceptibility candidate 2, microRNA-96-5p, synoviolin was a tumor suppressor, which inhibited the growth and metastasis of BC through the miR-96-5p/SYVN1 axis.

\section{Introduction}

Breast cancer (BC) is the leading cause of cancer-associated mortality in women worldwide, with $>1,000,000$ diagnosed cases annually (1). Although the diagnosis and treatment of BC has improved, the overall survival rate for patients with $\mathrm{BC}$, particularly for those with advanced stage, remains poor (2). Several molecules have been reported to serve essential roles in $\mathrm{BC}$ development and progression $(3,4)$. However, the precise regulatory mechanisms of these molecules remain poorly understood (5). Determining the molecular mechanisms that are associated with $\mathrm{BC}$ progression and metastasis is therefore imperative.

Long non-coding RNAs (lncRNAs), which are transcripts $>200$ nucleotides without a protein-coding capacity, have been demonstrated to be involved in the initiation, progression and metastasis of numerous types of cancer (6). IncRNA cancer susceptibility candidate 2 (CASC2) is a novel lncRNA located at chromosome 10q26, which has been identified as a tumor suppressor in multiple human malignancies $(7,8)$. CASC2 was reported to inhibit gastric cancer and hepatocellular carcinoma cell proliferation, migration and invasion through the suppression of the mitogen activated protein kinase signaling pathway, which is involved in the pathogenesis of various cancer types $(9,10)$. CASC2 was also revealed to suppress glioma cell and bladder cancer cell proliferation and metastasis through the inactivation of the Wnt/â-catenin pathway, which is a conserved molecular mechanism with an important role in multiple human malignancies $(11,12)$. In addition, CASC2 has been demonstrated to function as a competing endogenous RNA for microRNA and in turn modulates the expression 
of microRNA (miR) target genes (13). In colorectal cancer, CASC2 acts as a sponge of miR-18a to regulate the expression of protein inhibitor of activated STAT3 thereby inhibiting cancer growth in vitro and in vivo, and represents a biomarker for the diagnosis and therapeutic of colorectal cancer (14). CASC2 inhibits tumorigenesis in esophageal carcinoma by targeting miR-18a-5p, whereas it increases the sensitivity of prostate cancer cells to docetaxel by sponging miR-183 $(15,16)$. Zhang et al (17) reported that IncRNA CASC2 suppresses BC cell proliferation and metastasis through inactivation of the tumor growth factor- $\beta$ signaling pathway.

A number of studies have demonstrated that miR-96-5p is implicated in the regulation of proliferation, apoptosis, migration and invasion of several types of cancer (18-20). Serum concentrations of miR-96-5p are significantly upregulated in $\mathrm{BC}$ and are associated with a reduced survival rate of patients with BC (21). Furthermore, miR-96-5p expression is significantly elevated in BC tissues and cell lines compared with adjacent normal tissues and non-malignant breast epithelial cells, respectively $(22,23)$. The overexpression of miR-96-5p promotes the proliferation and migration of $\mathrm{BC}$ cells, while the inhibition of miR-96-5p leads to a decrease in cell viability and an increase in cell death $(24,25)$. miR-96-5p serves an oncogenic role in $\mathrm{BC}$ progression, but the regulatory mechanism requires further elucidation.

In the present study, IncRNA CASC2 expression was determined in $\mathrm{BC}$. The results demonstrated that the lncRNA CASC2 level was significantly decreased in BC tissues and cells compared with adjacent normal tissues and mammary epithelial cells, respecitively. Furthermore, the overexpression of CASC2 was identified to induce apoptosis and suppress the migration of BC cells. The upregulation of IncRNA CASC2 resulted in decreased miR-96-5p expression and increased synoviolin (SYVN1) expression. In combination, these results demonstrated that lncRNA CASC2 inhibited BC cell growth and metastasis through the regulation of the miR96-5p/SYVN1 signaling pathway.

\section{Methods and materials}

Patients and tissue samples. A total of 35 paired tissue samples of breast cancer and adjacent normal tissues were obtained from the Affiliated Hospital of Integrated Traditional Chinese and Western Medicine (Nanjing, China) between January 2016 and February 2017 from female patients with a median age of 52 years (age range, 31-69 years). A total of eight patients had stage I, 17 had stage II and 10 had stage III breast cancer at the time of the surgery. In addition, 19 (54.3\%) patients were negative and $16(45.7 \%)$ patients were positive for lymph node-metastasis, and 22 (62.9\%) patients were estrogen receptor-positive, 18 (51.4\%) patients were progesterone-positive, and 12 (34.3\%) patients were human epidermal growth factor receptor 2-positive. The pathological features and tumor stage were reviewed by two different experienced pathologists according to the World Health Organization Classification. Clinical information was obtained from patient charts and pathological reports. No patient had received radiotherapy or chemotherapy prior to surgical resection. Patients were excluded from the present study if they exhibited bilateral disease or pregnancy with the diagnosis of breast cancer. All the tissue samples were immediately frozen in liquid nitrogen and stored at $-80^{\circ} \mathrm{C}$ until total RNA and protein were extracted. The study on BC samples was approved and supervised by the Research Ethics Committee of Nanjing Medical University (Nanjing, China). Written informed consent was obtained from all patients.

Cell lines and cell culture. Human BC (MCF-7, MDA-MB-231) and mammary epithelial (MCF10A) cell lines were maintained at Nanjing Medical University (Nanjing, China). MCF10A cells were cultured in Dulbecco's modified Eagle's medium (DMEM)/F12 (3:1) supplemented with 10\% fetal bovine serum (FBS) (both from Thermo Fisher Scientific, Inc., Waltham, MA, USA), $20 \mathrm{ng} / \mathrm{ml}$ of epidermal growth factor, $0.5 \mu \mathrm{g} / \mathrm{ml}$ hydrocortisone, $10 \mu \mathrm{g} / \mathrm{ml}$ insulin, $50 \mathrm{U} / \mathrm{ml}$ penicillin and $50 \mu \mathrm{g} / \mathrm{ml}$ streptomycin. MCF7 and MDA-MB-231 cells were cultured in DMEM + GlutaMAX ${ }^{\mathrm{TM}}$ (Thermo Fisher Scientific, Inc., Waltham, MA, USA) containing 10\% FBS, $50 \mathrm{U} / \mathrm{ml}$ penicillin and $50 \mu \mathrm{g} / \mathrm{ml}$ streptomycin. All cells were cultured at $37^{\circ} \mathrm{C}$ in a humidified atmosphere containing $5 \% \mathrm{CO}_{2}(26)$.

Reverse transcription-quantitative polymerase chain reaction $(R T-q P C R)$. Total RNA was extracted from tissues and cells using TRIzol reagent (Thermo Fisher Scientific, Inc.) according to the manufacturer' s protocol. For each sample, $1 \mu \mathrm{g}$ of the total RNA was converted to cDNA using the High-Capacity cDNA Reverse Transcription kit (Thermo Fisher Scientific, Inc.) and performed at $45^{\circ} \mathrm{C}$ for $60 \mathrm{~min}$ followed by $70^{\circ} \mathrm{C}$ for $10 \mathrm{~min}$. The expression of CASC2, miR-96-5p, miR-183-5p, miR-182-5p, miR-155, miR-21, miR-31, miR-221 and miR-27a was measured by qPCR using a LightCycler480 II Sequence Detection system (Roche Diagnostics, Basel, Switzerland). The following primers were used: CASC2 forward, 5'-GCTGATCAGAGCACATTGGA-3' and reverse, 5'-ATAAAGGTGGCCACAACTGC-3'; SYVN1 forward, 5'-AACCCCTGGGACAACAAGG-3' and reverse, 5'-GCGAGACATGATGGCATCTG-3'; GAPDH forward, 5'-GGGAGCCAAAAGGGTCAT-3' and reverse, 5'-GAGTCC TTCCACGATACCAA-3'; miR-96-5p forward, 5'-TTTGGC ACTAGCACAT-3' and reverse, 5'-GAGCAGGCTGGAGAA-3'; miR-183-5p forward, 5'-CGCGGTATGGCACTGGTAGA-3' and reverse, 5'-AGTGCAGGGTCCGAGGTATTC-3'; miR-182-5p forward, 5'-TGCGGTTTGGCAATGGTAGAAC-3' and reverse, 5'-CCAGTGCAGGGTCCGAGGT-3'; miR-155 forward, 5'-GCG GTTAATGCTAATCGTGAT-3' and reverse, 5'-GTGCAGGGT CCGAGGT-3'; miR-21 forward, 5'-UAGCUUAUCAGACUGA UGUUGA-3' and reverse, 5'-CGAGGAAGAAGACGGAAG AAT-3'; miR-31 forward, 5'-GCGGCGGAGGCAAGATGCT GGC-3' and reverse, 5'-AGGCAAGATGCTGGCATAGCT-3'; miR-221 forward, 5'-CGAGCTACATTGTCTGCTGGGT-3' and reverse, 5'-GTGCAGGGTCCGAGGT-3'; miR-27a forward, 5'-TGTATTTTAGTCGTGGCGATA-3' and reverse, 5'-ATAACG ACTCACGCCTATAATC-3'; U6 forward, 5'-GTGCGTGTCGT GGAGTCG-3'andreverse,5'-AACGCTTCACGAATTTGCGT-3'. U6 and GAPDH were used as internal standards. The qPCR analysis was performed using the SYBR premix Ex Taq II kit (Takara Biotechnology, Co., Ltd., Dalian, China). The thermocycling conditions were as follows: $95^{\circ} \mathrm{C}$ for $30 \mathrm{sec}$; followed by 40 cycles at $95^{\circ} \mathrm{C}$ for $15 \mathrm{sec}, 57^{\circ} \mathrm{C}$ for $30 \mathrm{sec}$ and $72^{\circ} \mathrm{C}$ for $34 \mathrm{sec}$; and a final extension step at $72^{\circ} \mathrm{C}$ for $5 \mathrm{~min}$. Relative expression 
levels were analyzed using the $2^{-\Delta \Delta \mathrm{Cq}}$ method as previously described (27).

Western blot analysis. Total protein from BC cells were extracted in lysis buffer were lysed with ice-cold lysis buffer containing: $50 \mathrm{mmol} / \mathrm{l}$ Tris-HCl, pH 7.4; $1 \% \mathrm{NP}-40 ; 150 \mathrm{mmol} / \mathrm{l} \mathrm{NaCl}$; $1 \mathrm{mmol} / \mathrm{l}$ EDTA; $1 \mathrm{mmol} / \mathrm{l}$ phenylmethylsulfonyl fluoride; and complete proteinase inhibitor mixture (one tablet $/ 10 \mathrm{ml}$; Roche Molecular Biochemicals, Pleaston, CA, USA). The protein concentration was measured using a Bradford protein assay. Equal quantities of protein $(30 \mu \mathrm{g})$ were subjected to $10 \%$ SDS-PAGE and transferred onto polyvinylidene difluoride membranes. The membranes were blocked with $5 \%$ bovine serum albumin (Beyotime Institute of Biotechnology, Haimen, China) for $2 \mathrm{~h}$ at room temperature and incubated overnight at $4^{\circ} \mathrm{C}$ with primary antibodies. Western blot analysis was performed as previously described (26). Individual immunoblots were probed with a rabbit anti-SYVN1 (1:1,000; cat. no. AV43360; Merck KGaA, Darmstadt, Germany) and a mouse anti- $\beta$-actin antibody (1:3,000; cat. no. sc-517582; Santa Cruz Biotechnology, Inc., Dallas, TX, USA). The blots were incubated with horseradish peroxidase-conjuagated goat anti-rabbit IgG (cat. no. A0208) and goat anti-mouse IgG (cat. no. A0216) (both 1:1,000; Beyotime Institute of Biotechnology) secondary antibodies for $1 \mathrm{~h}$ at room temperature. The relative protein expression was determined using ImageJ V1.8.0 (National Institutes of Health, Bethesda, MD, USA) with $\beta$-actin used as the internal reference.

Plasmid construction. CASC2 cDNA coding sequence was amplified according to the full-length CASC2 sequence using the following primer pair: Forward, 5'-TGCATCAGACAG GAGTAGATG-3' and reverse, 5'-GCTATGCGCCAAGTTAA CAG-3'. PCR products were subcloned into a pcDNA3.1 vector (Thermo Fisher Scientific, Inc.). The plasmid was sequenced and confirmed to be correct. An empty pcDNA3.1 vector served as the negative control.

Cell viability assay. Cell viability was quantified using the Cell Counting Kit-8 (CCK-8; Dojindo Molecular Technologies, Inc., Kumamoto, Japan). Briefly, MCF-7 and MDA-MB-231 cells were seeded into a 48 -well plate $\left(6 \times 10^{3}\right.$ cells/well $)$ and were transfected with pcDNA3.1 or pcDNA3.1-CASC2 $(0.5 \mu \mathrm{g} /$ well). Following transfection for 24,48 and $72 \mathrm{~h}$, the CCK- 8 reagent was added to the culture wells, which were then incubated at $37^{\circ} \mathrm{C}$ for an additional $2 \mathrm{~h}$. The absorbance was determined at $450 \mathrm{~nm}$ using a microtiter plate reader. Experiments were performed in triplicate.

Cell apoptosis assay. Apoptosis was analyzed by flow cytometric analysis. The pcDNA3.1 or pcDNA3.1-CASC2transfected MCF-7 and MDA-MB-231 cells were cultured in 6-well plates for $48 \mathrm{~h}$. The cells were harvested by trypsinization. Following double staining with FITC-Annexin V and propidium iodide (BD Biosciences, San Jose, CA, USA) for $15 \mathrm{~min}$ at room temperature in the dark, the cells were analyzed using flow cytometry (BD FACScan ${ }^{\mathrm{TM}}$ system; BD Biosciences). Flow cytometry data were analyzed using Kaluza analysis software version 2.0 (Beckman Coulter, Inc., Brea, CA, USA). The assay was repeated in triplicate.
Invasion and migration assay. For the migration assays, $1 \times 10^{5}$ MDA-MB-231 cells in serum-free medium were placed into the upper chamber of a Transwell insert $(8-\mu \mathrm{m}$ pore size; Sigma-Aldrich; Merck KGaA). For the invasion assays, MDA-MB-231 cells in serum-free medium were placed into the upper chamber of an insert coated with Matrigel (Sigma-Aldrich; Merck KGaA). DMEM containing 10\% FBS was added to the lower chamber. Following incubation for $12 \mathrm{~h}$, the cells remaining on the upper membrane were removed using cotton wool. BC cells that had migrated or invaded through the membrane were fixed in $10 \%$ methanol for $15 \mathrm{~min}$ at room temperature, and then stained with crystal violet dye $(0.04 \%$ in $\mathrm{H}_{2} \mathrm{O} ; 100 \mu \mathrm{l}$ ) for $20 \mathrm{~min}$ at room temperature, counted using an inverted microscope and imaged (magnification, x200).

Pull-down assay with biotinylated lncRNA-CASC2 DNA probe. CASC2 and its antisense RNA were in vitro transcribed and biotin-labeled with the Biotin RNA Labeling mix and T7/SP6 RNA polymerase (both from Roche Diagnostics), and purified using a RNeasy Mini kit (Qiagen, Inc., Valencia, CA, USA) according to the manufacturer's protocol. The biotinylated lncRNA-CASC2 DNA probe was dissolved in binding and washing buffer, and incubated with Dynabeads M-280 streptavidin (Invitrogen; Thermo Fisher Scientific, Inc.) at room temperature for $10 \mathrm{~min}$ to generate probe-coated beads according to the manufacturer's protocol. Subsequently, MDA-MB-231 cell lysates were incubated with the probecoated beads, and the RNA complexes bound to these beads were eluted and extracted for RT-qPCR as aforementioned.

Pull-down assay with biotinylated miR-96-5p. MDA-MB-231 cells were transiently transfected with biotinylated miR-96-5p, miR-96-5p-mutant (Mut) and negative control (Guangzhou RiboBio Co., Ltd., Guangzhou, China), then harvested and lysed $48 \mathrm{~h}$ after transfection. Subsequently, $50 \mu \mathrm{l}$ of the samples were aliquoted for input. The remaining lysates were incubated with Dynabeads M-280 streptavidin according to the manufacturer' s protocol. In brief, the washed beads were treated with RNase-free solutions and incubated with an equal volume of biotinylated miR-96-5p for $10 \mathrm{~min}$ at room temperature in binding and washing buffer on a rotator. Next, the beads with the immobilized miR-96-5p fragment were incubated with $10 \mathrm{mM}$ EDTA (pH 8.2) with $95 \%$ formamide at $65^{\circ} \mathrm{C}$ for $5 \mathrm{~min}$. The bound RNAs were purified using TRIzol for RT-qPCR as aforementioned.

miRNA target prediction. Prediction of the miR-96-5p targets was performed using two publicly available algorithms: TargetScan6.2 (http://www.targetscan.org/) and miRanda (http://www.microrna. org/).

Luciferase reporter assays. To construct the reporter vector, one fragment of SYVN1 3'-untranslated region (3'-UTR) [wildtype (WT) or mutant (MUT), respectively] and the fragment of CASC2 containing predicted miR-96-5p binding site, or the fragment of miR-96-5p, containing predicted CASC 2 binding site were separately amplified, and fused to a modified pcDNA3.1 vector containing a luciferase gene, which was cloned into upstream of cloning sites. The luciferase assay was performed using the Dual-Luciferase ${ }^{\circledR}$ Reporter assay system 

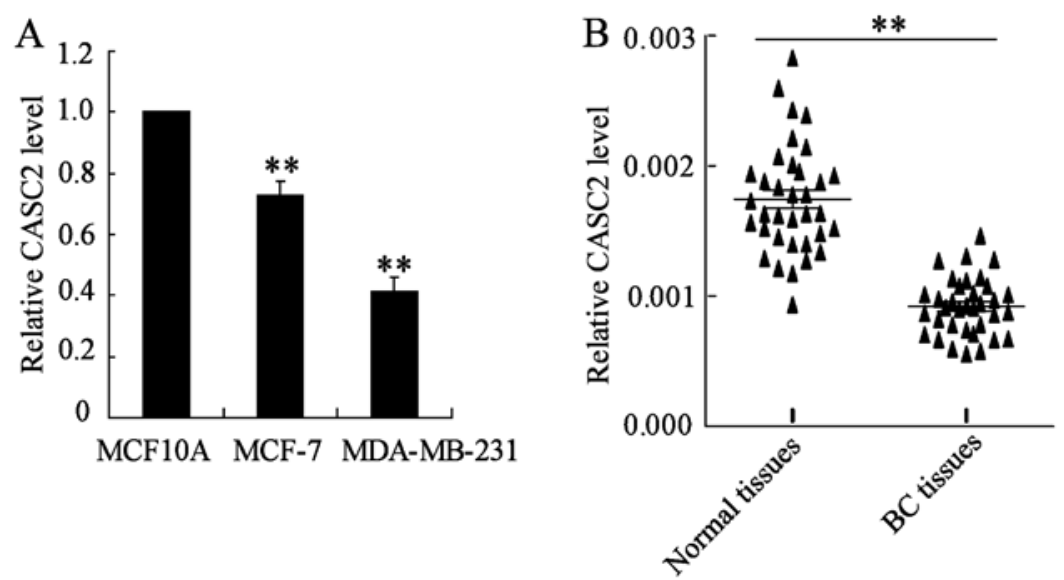

Figure 1. 1ncRNA CASC2 expression is downregulated in BC tissues and cell lines. (A) lncRNA CASC2 expression was determined using RT-qPCR in MCF10A, MCF-7 and MDA-MB-231 cells. (B) lncRNA CASC2 expression was determined by RT-qPCR in 35 pairs of BC and matched adjacent normal tissue samples. ${ }^{* *} \mathrm{P}<0.01$, compared with normal tissues or MCF10A cells. IncRNA, long non-coding RNAs; CASC2, lncRNA cancer susceptibility candidate 2; RT-qPCR, reverse transcription-quantitative polymerase chain reaction; BC, breast cancer.

(Promega Corporation, Madison, WI, USA). Briefly, BC cells were co-transfected with 100 pmol miR-96-5p mimics or 100 pmol pre-negative control (NC) and $0.5 \mu \mathrm{g}$ pMIR-reporter luciferase vector containing a specific sequence of WT or MUT CASC2 or SYVN1 fragment, using Lipofectamine 2000 (Thermo Fisher Scientific, Inc.). The miR-96-5p mimics, 5'-UUUGGCACUAGCACAUUUUUGCU-3', and NC, 5'-UUC UCCGAACGUGUCACGUTT-3' were obtained from Shanghai GenePharma Co., Ltd. (Shanghai, China). The luciferase activity was measured $48 \mathrm{~h}$ post-transfection. The relative luciferase activity was normalized to the Renilla luciferase activity.

Animal tumor model. Female athymic 6-weeks-old nude mice (mean weight, 10-12 g; n=7 mice/group) were purchased from the Shanghai Laboratory Animal Centre (Chinese Academy of Sciences, Shanghai, China) and maintained in cage housing under specific pathogen-free conditions with free access to food and water. Cultured MDA-MB-231 cells transfected with enhanced green fluorescent protein (EGFP) (Len-GFP, 10-fold multiplicity of infection virus particle concentration) or EGFP-tagged CASC2 lentivirus (Len-CASC2, 10-fold multiplicity of infection virus particle concentration) in the presence of $4 \mu \mathrm{g} / \mathrm{ml}$ polybrene (Sigma-Aldrich; Merck KGaA) were harvested from 6-well plates and resuspended in $0.2 \mathrm{ml}$ PBS at $5 \times 10^{7}$ cells $/ \mathrm{ml}\left(1 \times 10^{6}\right.$ cells/mouse). Len-GFP and Len-CASC 2 were obtained from Shanghai GenePharma Co., Ltd. Cells were injected into the right or the left flank region of the mice to generate the orthotopic model. Tumor volumes $\left(\mathrm{mm}^{3}\right)$ in mice were measured with a slide caliper every 4 days according to the formula: $1 / 2 \mathrm{x}$ width ${ }^{2} \mathrm{x}$ length. Animals were treated humanely, using approved procedures in accordance with the guidelines of the Institutional Animal Care and Use Committee at Nanjing Medical University. The study was approved by the Experimental Animal Ethics Committee of Nanjing Medical University.

Statistical analysis. Statistical analysis was performed using SPSS 18.0 statistical analysis software (SPSS, Inc., Chicago, IL, USA). Comparisons between two groups were performed using the Student's t-test and comparisons among multiple groups using one-way analysis of variance with Tukey's post hoc test. The correlation between CASC 2 and miR-96-5p expression was analyzed using the Pearson correlation analysis. Data are expressed as the mean \pm standard deviation. $\mathrm{P}<0.05$ was considered to indicate a statistically significant difference. All experiments were repeated triplicate.

\section{Results}

IncRNA CASC2 expression is downregulated in BC tissues and cell lines. CASC 2 expression was determined in MCF10A, MCF-7 and MDA-MB-231 cells using RT-qPCR. The results demonstrated that the CASC2 expression level was significantly decreased in $\mathrm{BC}$ cells compared with the mammary epithelial MCF10A cells (Fig. 1A). In addition, the expression of 1 ncRNA CASC 2 in $\mathrm{BC}$ and matched adjacent normal tissue samples was measured. As shown in Fig. 1B, the CASC2 expression level was significantly downregulated in $\mathrm{BC}$ tissues, as compared with adjacent normal tissues.

CASC2 upregulation inhibits $B C$ cell viability, migration and invasion. To explore the role of CASC 2 in $\mathrm{BC}$ cells, CASC2 expression was increased through the transfection of pcDNA3.1-CASC2 in MDA-MB-231 and MCF-7 cells. The results revealed that pcDNA3.1-CASC2 significantly increased the expression of CASC2 (Fig. 2A). Next, the effect of pcDNA3.1-CASC2 on the growth of MDA-MB-231 and MCF-7 cells was investigated. The overexpression of CASC2 led to a decrease of cell growth in a time-dependent manner (Fig. 2B). In addition, the effects of CASC2 on the apoptosis of $\mathrm{BC}$ cells were measured by flow cytometry. As shown in Fig. 2C, the rate of apoptotic cells in pcDNA3.1and pcDNA3.1-CASC2-transfected MDA-MB-231 cells was 5.1 and $21 \%$, respectively. Similar results were observed in MCF-7 cells. However, pcDNA3.1-CASC2 had no significant effect on cell cycle distribution (data not shown). These data indicated that the decrease in the number of $\mathrm{BC}$ cells upon pcDNA3.1-CASC2 transfection was caused by apoptosis.

Next, migration and invasion assays were performed in MDA-MB-231 cells transfected with pcDNA3.1-CASC2. The 

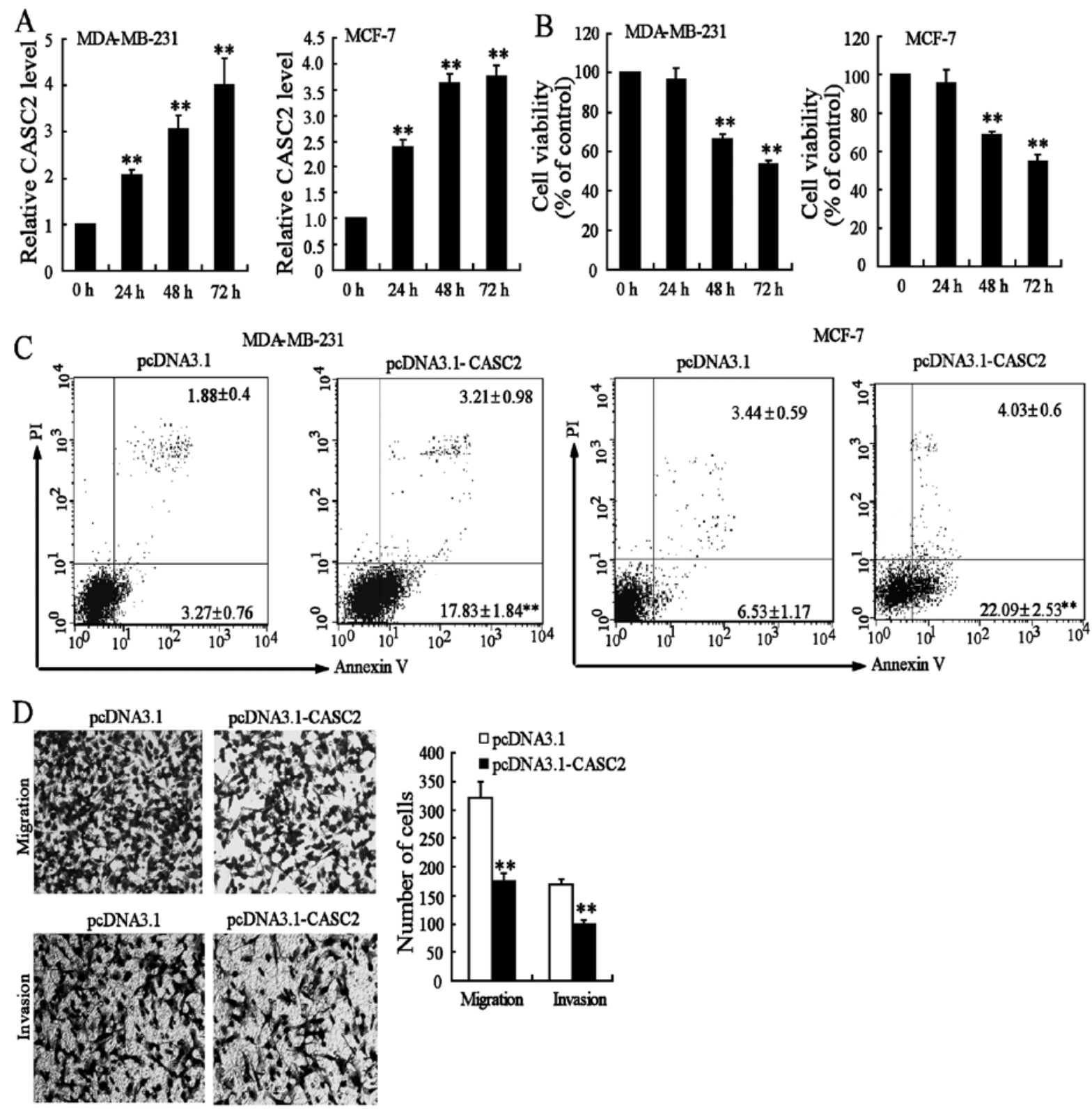

Figure 2. CASC2 upregulation inhibits BC cell viability, migration and invasion. MDA-MB-231 and MCF-7 cells were transfected with pcDNA3.1 or pcDNA3.1-CASC2 for different time periods and, (A) CASC2 expression and (B) cell viability was measured. (C) MDA-MB-231 and MCF-7 cells were transfected with pcDNA3.1 or pcDNA3.1-CASC2 for $48 \mathrm{~h}$ and cell apoptosis was determined. (D) MDA-MB-231 cells were transfected with pcDNA3.1 or pcDNA3.1-CASC2 for $48 \mathrm{~h}$, cell migration and invasion was determined. ${ }^{* *} \mathrm{P}<0.01$, compared with pcDNA3.1. CASC2, long non-coding RNAs cancer susceptibility candidate $2 ; \mathrm{BC}$, breast cancer.

results demonstrated that the overexpression of CASC2 significantly decreased migration and invasion in MDA-MB-231 cells, when compared with the controls (Fig. 2D). These results indicated that CASC2 may act as a tumor suppressor through the promotion of cell apoptosis and the suppression of cell migration and invasion in $\mathrm{BC}$.

CASC2 overexpression inhibits $B C$ growth in vivo. To further explore the effect of CASC2 overexpression on $\mathrm{BC}$ growth in vivo, an animal tumor model was established using MDA-MB-231 cells transfected with Len-GFP or Len-CASC2. The growth of the $\mathrm{BC}$ xenograft was significantly inhibited in mice treated with Len-CASC2, as compared with mice treated with Len-GFP(Fig.3A).Themean tumor weightinLen-CASC2-treated $\mathrm{BC}$ xenografts was significantly reduced compared with that in the Len-GFP group (53.33 \pm 5.5 vs. $113.33 \pm 13.41 \mathrm{mg} ; \mathrm{P}<0.01$; Fig. 3B). Therefore, the present data demonstrated that CASC2 overexpression inhibited $\mathrm{BC}$ development in vivo.

CASC2 functions as a miR-96-5p sponge in BC cells. lncRNA CASC2 may function as a competing endogenous RNA for microRNA due to sequence complementarity and in turn may regulate the expression of microRNA target genes (13-15). In the present study, the association between CASC2 and miR-96-5p expression in 35 BC tissues was explored, with the results revealed a significantly negative correlation between CASC2 and miR-96-5p expression levels $(\mathrm{r}=-0.825$; $\mathrm{P}<0.001$; Fig. 4A). Furthermore, overexpression of CASC2 was demonstrated to significantly inhibit miR-96-5p expression in MDA-MB-231 cells (Fig. 4B). A total of seven 

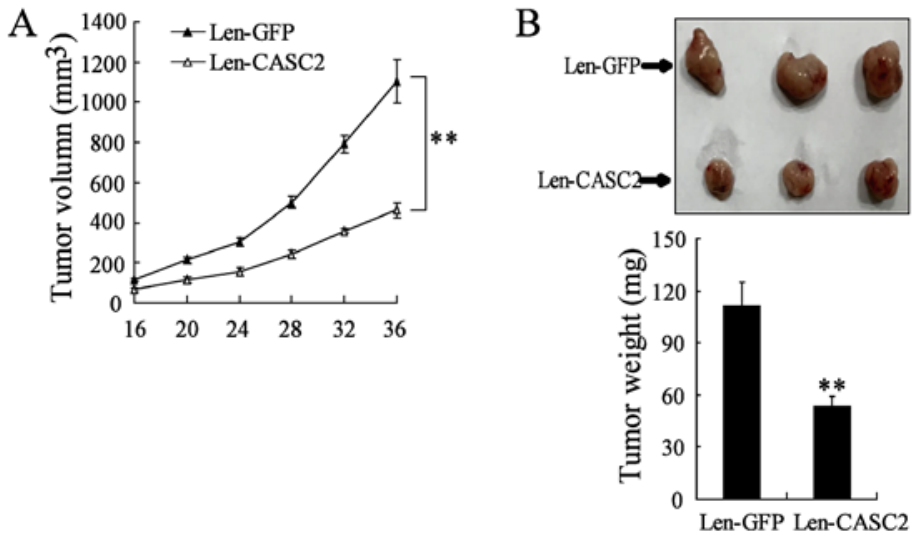

Figure 3. CASC2 over-expression inhibited BC growth in vivo. MDA-MB-231 cells transfected with CASC2 lentivirus (Len-CASC2) or control vector lentivirus (Len-GFP) were injected into the right and left flank of nude mice, respectively. (A) Tumor sizes were monitored every 4 days. (B) After 36 days, images of the tumors were captured and tumor weights were measured. ${ }^{* *} \mathrm{P}<0.01$, compared with Len-GFP. CASC2, long non-coding RNAs cancer susceptibility candidate 2; BC, breast cancer; Len-CASC2, CASC2 lentivirus; Len-GFP, control vector lentivirus.
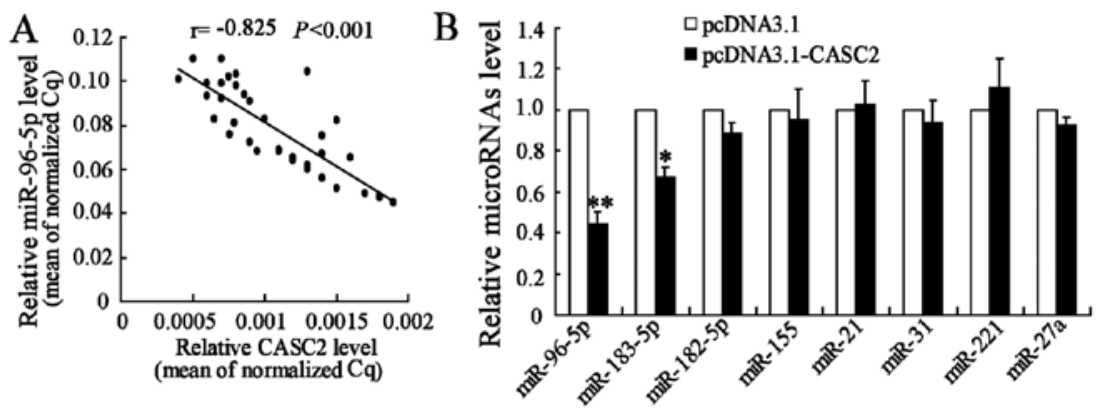 C Casc2-WT 5'-UCAaUauacugcugguggGchaA-3' miR-96-5p 3'-UCGUUUUUACACGAUCA--CGGUUU-5' CASC2-MUT5'-UCAAUAUACUGCUGGUGGAUUGGG-3'
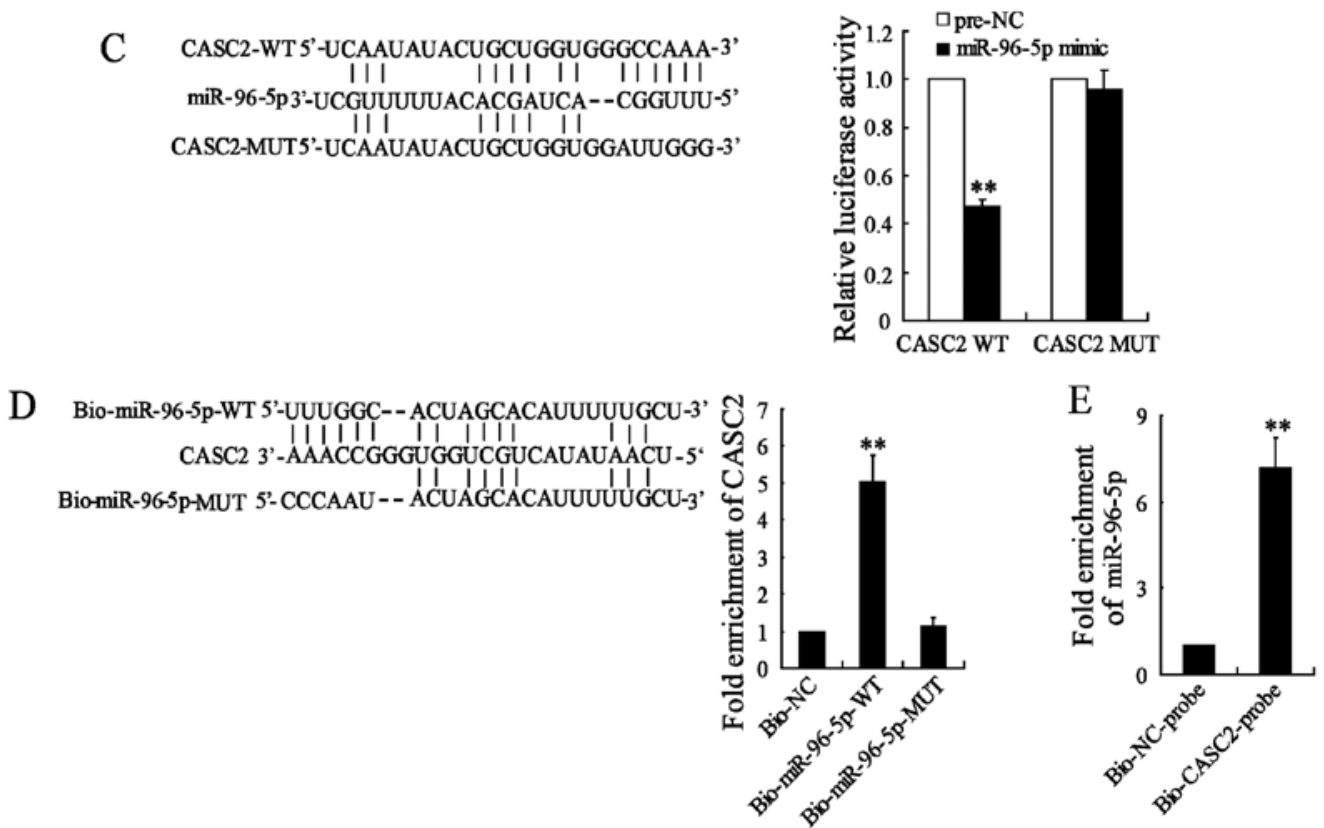

Figure 4. CASC2 functions as a miR-96-5p sponge in BC cells. (A) Correlation between the CASC2 and miR-96-5p expression in 35 BC tissues. (B) MDAMB-231 cells were transfected with pcDNA3.1 or pcDNA3.1-CASC2 for $48 \mathrm{~h}$ and the expression of miR-96-5p, miR-183, miR-182, miR-155, miR-21, miR-205, miR-221 and miR-27a was determined. (C) Sequence alignment of miR-96-5p with the putative binding sites within the WT regions of CASC2. MDA-MB-231 cells were co-transfected with a miR-96-5p mimic and CASC2-WT vector or CASC2-MUT vector for $48 \mathrm{~h}$ and the luciferase activity was measured. (D) The WT and mutated forms of the miR-96-5p sequence are shown. Detection of CASC2 using RT-qPCR in the sample pulled down by biotinylated miR-96-5p. (E) Detection of miR-96-5p using RT-qPCR in the sample pulled down by biotinylated CASC2 probe. " $\mathrm{P}<0.05$ and ${ }^{* *} \mathrm{P}<0.01$, compared with pcDNA3.1, Pre-NC or Bio-NC. CASC2, long non-coding RNAs cancer susceptibility candidate 2; BC, breast cancer; WT, wild type; MUT, mutant; NC, negative control; RT-qPCR, reverse transcription-quantitative polymerase chain reaction; UTR, untranslated region; miR, microRNA.

other miRNAs (miR-183-5p, miR-182-5p, miR-155, miR-21, miR-31, miR-221 and miR-27a) were also measured, which act as oncogenes in $\mathrm{BC}(28)$, and were predicted to be likely downstream targets of CASC2 from the database (LncBase 


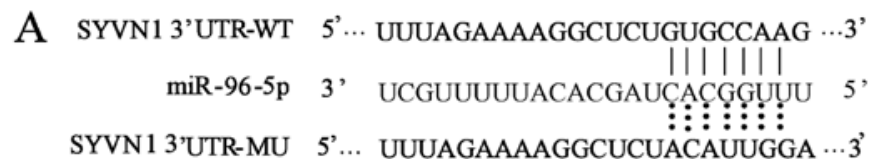

B

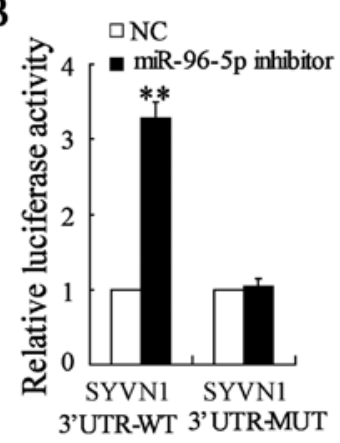

$\mathrm{C}$

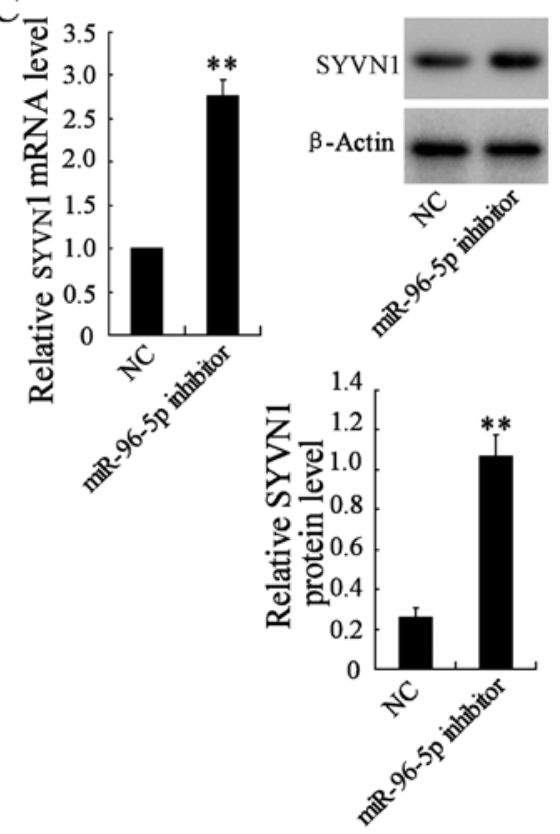

Figure 5. miR-96-5p directly targets SYVN1 in BC cells. (A) The 3'-UTR of SYVN1 harbored an miR-96-5p cognate site. (B) MDA-MB-231 cells were co-transfected with miR-96-5p inhibitor and WT or MUT SYVN1 3'-UTR for $48 \mathrm{~h}$ and the luciferase activity was determined. (C) MDA-MB-231 cells were transfected with NC or miR-96-5p inhibitor for $48 \mathrm{~h}$ and the mRNA and protein levels of SYVN1 were measured. ** $<<0.01$, compared with NC. BC, breast cancer; WT, wild type; MUT, mutant; NC, negative control; UTR, untranslated region; miR, microRNA; SYVN1, synoviolin.

Predicted V.2, http://www.microrna.gr/LncBase). The results revealed that the inhibitory effect of CASC2 on the expression of these seven miRNAs was lower, compared with that on miR-96-5p (Fig. 4B).

The database revealed that there were binding sites between CASC2 and miR-96-5p. The luciferase reporter assay demonstrated that the overexpression of miR-96-5p significantly decreased CASC2-WT activity, while it had no significant effect on CASC2-MUT (Fig. 4C). In addition, CASC2 was pulled down by miR-96-5p, but the mutations resulted in the inability of miR-96-5p to pull down CASC2 (Fig. 4D), which suggested that the recognition of miR-96-5p to CASC2 was in a sequence-specific manner. It was also observed that CASC2 pulled down miR-96-5p (Fig. 4E). These results revealed that CASC2 functioned as a miR-96-5p sponge in BC cells.

miR-96-5p directly targets SYVN1 in BC cells. It was demonstrated that miR-96-5p served an oncogenic role in BC. Our previous study reported that the overexpression of SYVN1 inhibited the growth, migration and invasion of BC cells in vitro and in vivo (26). TargetScan and miRanda revealed that the 3'-UTR of SYVN1 contained the complementary site for the seed region of miR-96-5p (Fig. 5A). Further examination demonstrated that miR-96-5p inhibition significantly increased the SYVN1 3'-UTR activity, which was not observed for the mutant SYVN1 3'-UTR activity (Fig. 5B). In addition, miR-96-5p inhibition significantly elevated the mRNA and protein expression of SYVN1 in MDA-MB-231 cells compared with the negative control (Fig. 5C). These data indicated that miR-96-5p targeted human SYVN1 by directly binding to the predicted sites in 3'-UTR of SYVN1 mRNA.

CASC2 regulates SYVN1 expression through modulating miR-96-5p. As CASC2 share regulatory miR-96-5p with SYVN1 mRNA, the possibility of CASC2 regulating SYVN1 in BC cells was explored. As shown in Fig. 6A, the overexpression of CASC2 significantly increased SYVN1 mRNA and protein levels in MDA-MB-231 cells compared with the control cells. In addition, the upregulation of miR-96-5p upon pcDNA3.1-CASC2 transfection abrogated this increase (Fig. 6B). All these data suggested an important role of CASC2 in regulating SYVN1 by competitively binding miR-96-5p.

CASC2 overexpression suppresses BC cell viability, migration and invasion via the miR-96-5p/SYVN1 axis. Whether the upregulation of CASC2 inhibited BC cell viability, migration and invasion through the miR-96-5p/SYVN1 axis was investigated. Notably, miR-96-5p overexpression inhibited apoptosis, and promoted migration and invasion of CASC2-overexpressing MDA-MB-231 cells compared with cells with CASC2 overexpression only (Fig. 7A and B). Furthermore, the restoration of SYVN1 abrogated the promoting effects of miR-96-5p on viability, migration and invasion of MDA-MB-231 cells (Fig. 7C and D). Thus, it was demonstrated that CASC2 suppressed BC cell viability, migration and invasion via the miR-96-5p/SYVN1 axis. 

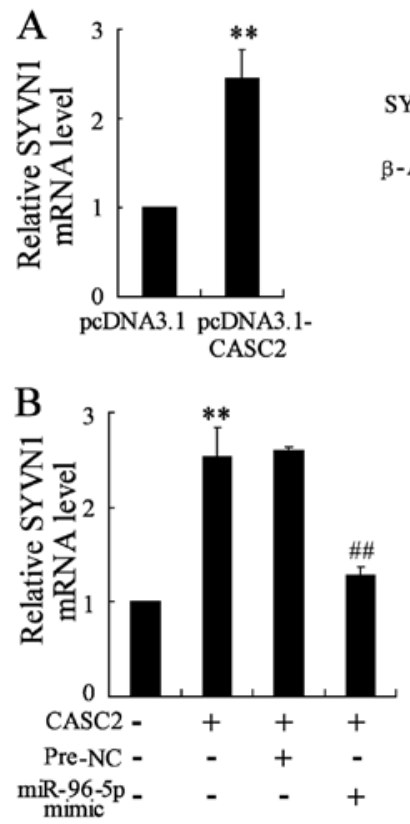
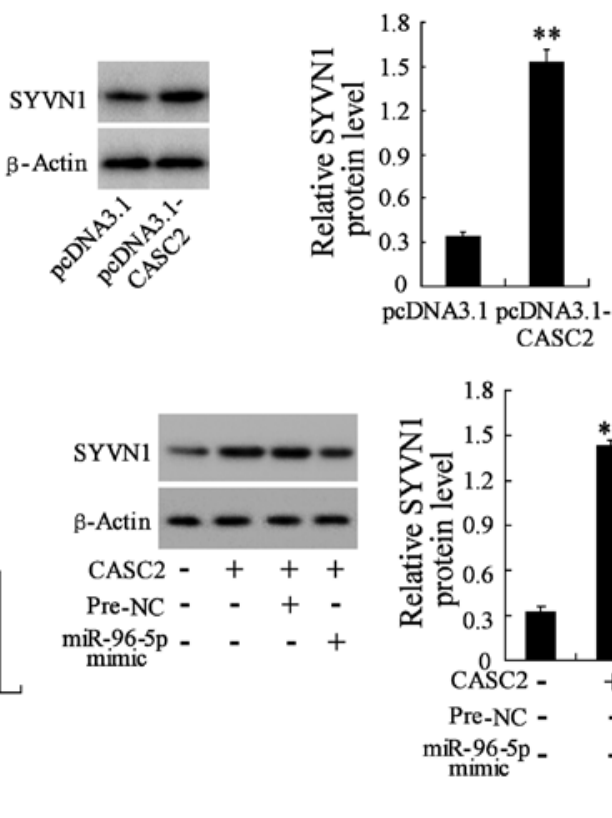

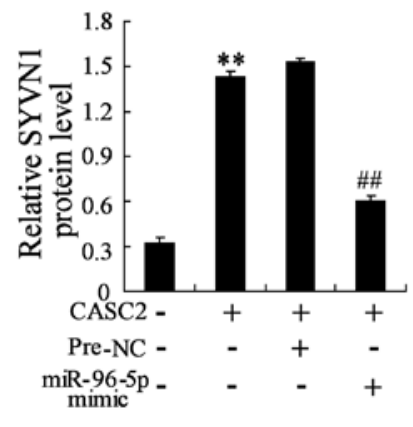

Figure 6. CASC2 regulates the SYVN1 expression through modulating miR-96-5p. (A) MDA-MB-231 cells were transfected with pcDNA3.1 or pcDNA3.1CASC2 for $48 \mathrm{~h}$ and the mRNA and protein levels of SYVN1 was determined. (B) MDA-MB-231 cells were transfected with pcDNA3.1-CASC2 (CASC2) and miR-96-5p mimic, and the mRNA and protein of SYVN1 expression was determined. ${ }^{* *} \mathrm{P}<0.01$, compared with pcDNA3.1; ${ }^{\# *} \mathrm{P}<0.01$, compared with pcDNA3.1-CASC2 + Pre-NC. CASC2, long non-coding RNAs cancer susceptibility candidate 2; NC, negative control; miR, microRNA; SYVN1, synoviolin.
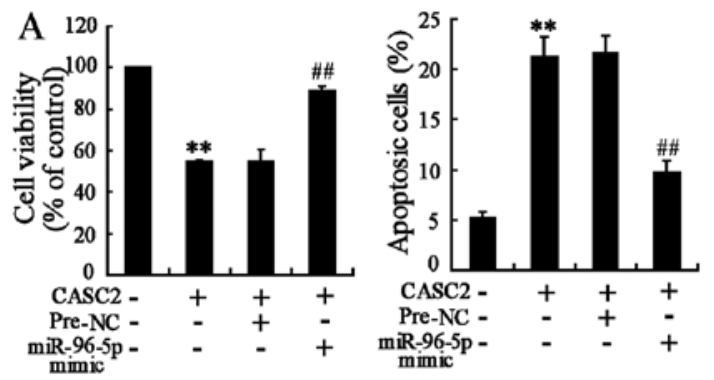
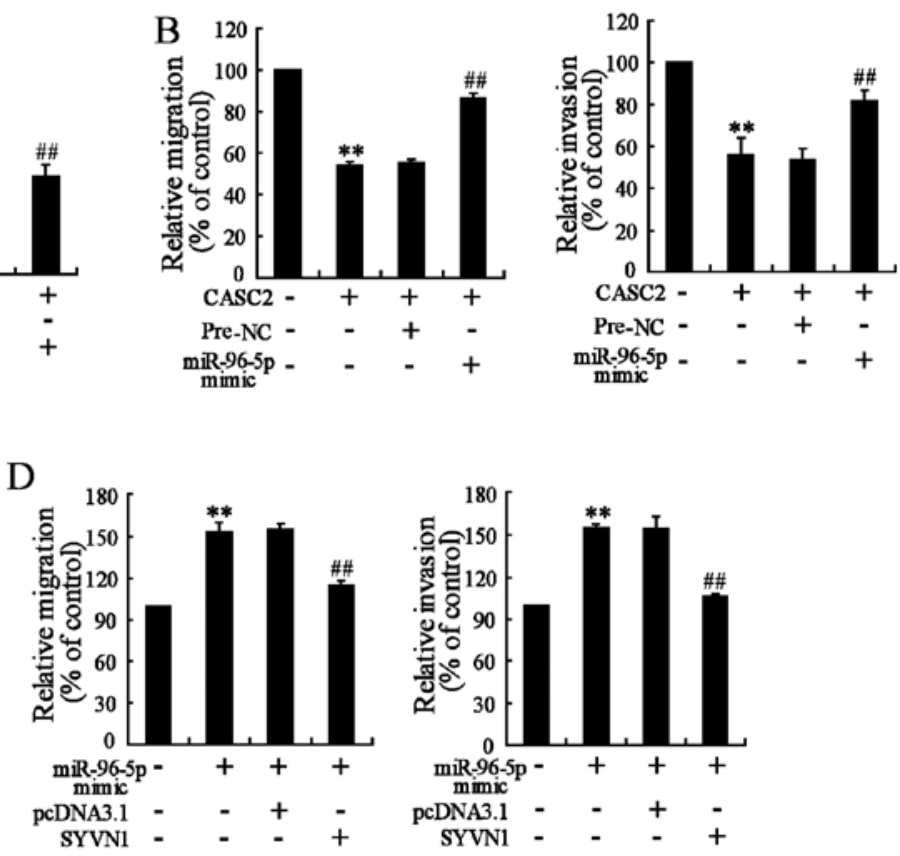

Figure 7. The CASC2 overexpression suppresses BC cell viability, migration and invasion through the miR-96-5p/SYVN1 axis. MDA-MB-231 cells were transfected with pcDNA3.1-CASC2 (CASC2) and miR-96-5p mimic for $48 \mathrm{~h}$, and then the (A) cell viability and cell apoptosis, and (B) cell migration and invasion were determined. MDA-MB-231 cells were transfected with an miR-96-5p mimic and pcDNA3.1-SYVN1 (SYVN1) for $48 \mathrm{~h}$, and the (C) cell viability, and (D) cell migration and cell invasion was determined. ${ }^{* *} \mathrm{P}<0.01$, compared with pcDNA3.1 or Pre-NC; ${ }^{\# \#} \mathrm{P}<0.01$, compared with pcDNA3.1-SYVN1 + Pre-NC or miR-96-5p mimic + pcDNA3.1. CASC2, long non-coding RNAs cancer susceptibility candidate 2; NC, negative control; miR, microRNA; SYVN1, synoviolin.

\section{Discussion}

Several studies have reported that a number of lncRNAs serve an important role in the occurrence and development of tumors in humans (29). IncRNA CASC2 has been revealed to be involved in the proliferation and metastasis of various types of cancer, including gastric, bladder and colorectal cancer, hepatocellular carcinoma, and glioma (6). However, the role and mechanism of lncRNA CASC 2 in BC remain unclear. The findings of the present study suggested that downregulation of lncRNA CASC2 in BC tissues and cell lines may act as a tumor suppressor. The mechanism revealed that dysregulation of the CASC2/miR-96-5p/SYVN1 axis contributed to BC cell proliferation, migration and invasion. 
Low expression of CASC2 has been reported in several human malignancies (9-12). In the present study, CASC2 expression was significantly inhibited in BC tissues and cell lines compared with adjacent normal tissues and nonmalignant breast epithelial cells, respectively. Furthermore, low expression of CASC2 was demonstrated to be significantly associated with the TNM stage, differentiation grade and lymph node metastasis of BC. In addition, CASC2 overexpression significantly inhibited $\mathrm{BC}$ growth in vitro and in vivo, and induced apoptosis in BC cells. CASC2 overexpression also inhibited the migration and invasion of $\mathrm{BC}$ cells. These findings demonstrated that CASC2 acted as a tumor suppressor in BC tissues and cells, which was in accordance with the function of CASC2 identified in other cancer tissues (30).

lncRNA CASC2 may function as a competing endogenous RNA by sponging miR-18a, miR-193a-5p or miR-183 and subsequently regulating the expression of the target genes of these microRNA (14-16,31). In the present study, a significantly negative association was identified between the CASC 2 and miR-96-5p expression levels in BC tissues. Furthermore, the CASC2 overexpression significantly decreased the expression level of miR-96-5p, but had a minimal effect on the expression of miR-183-5p, miR-182-5p, miR-155, miR-21, miR-31, miR-221 and miR-27a in BC cells. Since lncRNAs contain microRNA responsive elements and act as microRNA sponges to downregulate microRNA expression, it was observed that CASC2 may directly interact with miR-96-5p and downregulate its expression in $\mathrm{BC}$ cells. In addition, the present data indicated that miR-96-5p overexpression may reverse the anticancer effects of CASC 2 overexpression on BC cell growth, migration and invasion. Therefore, lncRNA CASC2 acted as a tumor suppressor in BC tissues and cell lines, at least partly, through the inhibition of miR-96-5p.

In $\mathrm{BC}, \mathrm{miR}-96-5 \mathrm{p}$ is a positive regulator of the proliferation and metastasis processes, in which the miR-183-5p expression is increased (22-25). In our previous study, SYVN1 was revealed to be a tumor suppressor in $\mathrm{BC}$ (26). Bioinformatics analysis revealed that SYVN1 may be a downstream target of miR-96-5p. Further investigations confirmed that miR-96-5p targeted human SYVN1 by directly binding to the predicted sites in the 3'-UTR of SYVN1 mRNA. Subsequently, it was observes that CASC2 positively regulated SYVN1 expression via targeting miR-96-5p in BC cells. The restoration of SYVN1 abrogated the promoter effects of miR-96-5p on the migration, invasion and viability of $\mathrm{BC}$ cells.

In conclusion, CASC 2 may inhibit cell migration, invasion and viability via the CASC2/miR-96-5p/SYVN1 axis in BC. Therefore, the suppressive effect of CASC2 on BC development indicated that IncRNA-MIAT may be a potential therapeutic target in $\mathrm{BC}$.

\section{Acknowledgements}

Not applicable.

\section{Funding}

Thepresentstudy was supported by the NationalKey Researchand Development Program of China (grant no. 2016YFC0905900),
The '333' Talent Project of Jiangsu Province [grant no. 4(2016)], The National Key Clinical Specialist Construction Programs of China [grant no. 544 (2013)], The Natural Science Foundation of Jiangsu Province (grant no. BK20151579) and The National Natural Science Foundation of China (grant nos. 81570779 and HG13-06).

\section{Availability of data and materials}

The datasets used and/or analyzed during the current study are available from the corresponding author on reasonable request.

\section{Authors' contributions}

DS and JT designed the study and wrote the manuscript. ZG, ML, HW, HL, JW and WZ performed the experiments. XL analyzed the data. All authors reviewed and approved the final manuscript.

\section{Ethics approval and consent to participate}

The study on BC samples was approved and supervised by the Research Ethics Committee of Nanjing Medical University (Nanjing, China). Written informed consent was obtained from all patients. Animals were treated humanely, using approved procedures in accordance with the guidelines of the Institutional Animal Care and Use Committee at Nanjing Medical University. The study was approved by the Experimental Animal Ethics Committee of Nanjing Medical University.

\section{Patient consent for publication}

Not applicable.

\section{Competing interests}

The authors declare that they have no competing interests.

\section{References}

1. Zduriencikova M, Gronesova P, Cholujova D and Sedlak J: Potential biomarkers of exosomal cargo in endocrine signaling. Endocr Regul 49: 141-150, 2015.

2. Paracha N, Thuresson PO, Moreno SG and MacGilchrist KS : Health state utility values in locally advanced and metastatic breast cancer by treatment line: A systematic review. Expert Rev Pharmacoecon Outcomes Res 16: 549-559, 2016.

3. Osborne C, Wilson P and Tripathy D: Oncogenes and tumor suppressor genes in breast cancer: Potential diagnostic and therapeutic applications. Oncologist 9: 361-377, 2004.

4. Logan GJ, Dabbs DJ, Lucas PC, Jankowitz RC, Brown DD, Clark BZ, Oesterreich S and McAuliffe PF: Molecular drivers of lobular carcinoma in situ. Breast Cancer Res 17: 76, 2015.

5. Panno ML, Naimo GD, Spina E, Andò S and Mauro L: Different molecular signaling sustaining adiponectin action in breast cancer. Curr Opin Pharmacol 31: 1-7, 2016.

6. Sun W, Yang Y, Xu C and Guo J: Regulatory mechanisms of long noncoding RNAs on gene expression in cancers. Cancer Genet 216-217: 105-110, 2017.

7. Baldinu P, Cossu A, Manca A, Satta MP, Sini MC, Palomba G, Dessole S, Cherchi P, Mara L, Tanda F, et al: CASC2a gene is down-regulated in endometrial cancer. Anticancer Res 27: $235-243,2007$ 
8. Palmieri G, Paliogiannis P, Sini MC, Manca A, Palomba G, Doneddu V, Tanda F, Pascale MR and Cossu A: Long non-coding RNA CASC2 in human cancer. Crit Rev Oncol Hematol 111: 31-38, 2017

9. Li P, Xue WJ, Feng Y and Mao QS: Long non-coding RNA CASC2 suppresses the proliferation of gastric cancer cells by regulating the MAPK signaling pathway. Am J Transl Res 8: $3522-3529,2016$

10. Gan Y, Han N, He X, Yu J, Zhang M, Zhou Y, Liang H, Deng J, Zheng Y, Ge W, et al: Long non-coding RNA CASC2 regulates cell biological behaviour through the MAPK signalling pathway in hepatocellular carcinoma. Tumour Biol 39: 1010428317706229 , 2017.

11. Pei Z, Du X, Song Y, Fan L, Li F, Gao Y, Wu R, Chen Y, Li W, Zhou $\mathrm{H}$, et al: Down-regulation of IncRNA CASC2 promotes cell proliferation and metastasis of bladder cancer by activation of the Wnt/ $\beta$-catenin signaling pathway. Oncotarget 8: 18145-18153, 2017.

12. Wang R, Li Y, Zhu G, Tian B, Zeng W, Yang Y and Li Z: Long noncoding RNA CASC2 predicts the prognosis of glioma patients and functions as a suppressor for gliomas by suppressing Wnt/ $\beta$-catenin signaling pathway. Neuropsychiatr Dis Treat 13 1805-1813, 2017.

13. Liao Y, Shen L, Zhao H, Liu Q, Fu J, Guo Y, Peng R and Cheng L: lncRNA CASC2 interacts with miR-181a to modulate glioma growth and resistance to TMZ through PTEN pathway. J Cell Biochem 118: 1889-1899, 2017

14. Huang G, Wu X, Li S, Xu X, Zhu H and Chen X: The long noncoding RNA CASC2 functions as a competing endogenous RNA by sponging miR-18a in colorectal cancer. Sci Rep 6: 26524, 2016.

15. Zhang W, He W, Gao J, Wang Y, Zang W, Dong Z and Zhao G RETRACTED: The long noncoding RNA CASC2 inhibits tumorigenesis through modulating the expression of PTEN by targeting miR-18a-5p in esophageal carcinoma. Exp Cell Res 361: 30-38, 2017.

16. Gao W, Lin S, Cheng C, Zhu A, Hu Y, Shi Z, Zhang X and Hong Z: Long non-coding RNA CASC2 regulates Sprouty2 via functioning as a competing endogenous RNA for miR-183 to modulate the sensitivity of prostate cancer cells to docetaxel. Arch Biochem Biophys: Jan 23, 2018 (Epub ahead of print). doi: 10.1016/j.abb.2018.01.013.

17. Zhang Y, Zhu M, Sun Y, Li W, Wang Y and Yu W: Up-regulation of lncRNA CASC2 suppresses cell proliferation and metastasis of breast cancer via inactivating of the TGF- $\beta$ signaling pathway. Oncol Res: Mar 9, 2018 (Epub ahead of print). doi: 10.3727/0965 $04018 X 15199531937158$.

18. Li C, Du X, Tai S, Zhong X, Wang Z, Hu Z, Zhang L, Kang P, Ji D, Jiang X, et al: GPC1 regulated by miR-96-5p, rather than miR-182-5p, in inhibition of pancreatic carcinoma cell proliferation. Int J Mol Sci 15: 6314-6327, 2014.
19. Ress AL, Stiegelbauer V, Winter E, Schwarzenbacher D Kiesslich T, Lax S, Jahn S, Deutsch A, Bauernhofer T, Ling H, et al: MiR-96-5p influences cellular growth and is associated with poor survival in colorectal cancer patients. Mol Carcinog 54: $1442-1450,2015$

20. Assal RA, El Tayebi HM, Hosny KA, Esmat G and Abdelaziz AI: A pleiotropic effect of the single clustered hepatic metastamiRs miR-96-5p and miR-182-5p on insulin-like growth factor II, insulin-like growth factor-1 receptor and insulin-like growth factor-binding protein-3 in hepatocellular carcinoma. Mol Med Rep 12: 645-650, 2015.

21. Zhang K, Wang YW, Wang YY, Song Y, Zhu J, Si PC and Ma R: Identification of microRNA biomarkers in the blood of breast cancer patients based on microRNA profiling. Gene 619: 10-20, 2017.

22. Zhang J, Kong X, Li J, Luo Q, Li X, Shen L, Chen L and Fang L: miR-96 promotes tumor proliferation and invasion by targeting RECK in breast cancer. Oncol Rep 31: 1357-1363, 2014.

23. Hong Y, Liang H, Uzair-Ur-Rehman, Wang Y, Zhang W, Zhou Y, Chen S, Yu M, Cui S, Liu M, et al: miR-96 promotes cell proliferation, migration and invasion by targeting PTPN9 in breast cancer. Sci Rep 6: 37421, 2016.

24. Shi Y, Zhao Y, Shao N, Ye R, Lin Y, Zhang N, Li W, Zhang Y and Wang S: Overexpression of microRNA-96-5p inhibits autophagy and apoptosis and enhances the proliferation, migration and invasiveness of human breast cancer cells. Oncol Lett 13: 4402-4412, 2017.

25. Xie W, Sun F, Chen L and Cao X: miR-96 promotes breast cancer metastasis by suppressing MTSS1. Oncol Lett 15: 3464-3471, 2018.

26. Xu YM, Wang HJ, Chen F, Guo WH, Wang YY, Li HY, Tang JH, Ding Y, Shen YC, Li M, et al: HRD1 suppresses the growth and metastasis of breast cancer cells by promoting IGF-1R degradation. Oncotarget 6: 42854-42867, 2015.

27. Livak KJ and Schmittgen TD: Analysis of relative gene expression data using real-time quantitative PCR and the 2(-Delta Delta $\mathrm{C}(\mathrm{T})$ ) method. Methods 25: 402-408, 2001

28. Lo PK, Wolfson B, Zhou X, Duru N, Gernapudi R and Zhou Q: Noncoding RNAs in breast cancer. Brief Funct Genomics 15: 200-221, 2016

29. Kondo Y, Shinjo K and Katsushima K: Long non-coding RNAs as an epigenetic regulator in human cancers. Cancer Sci 108: 1927-1933, 2017.

30. Lu L, Dai Z, Luo Q and Lv G: The long noncoding RNA cancer susceptibility candidate 2 inhibits tumor progression in osteosarcoma. Mol Med Rep 17: 1947-1953, 2018.

31. Jiang C, Shen F, Du J, Fang X, Li X, Su J, Wang X, Huang X and Liu Z: Upregulation of CASC2 sensitized glioma to temozolomide cytotoxicity through autophagy inhibition by sponging miR-193a-5p and regulating mTOR expression. Biomed Pharmacother 97: 844-850, 2018. 